\title{
EchoGéo
}

\section{Les migrants de Beyrouth}

Emplois à bon marché et zones refuges

\section{Agnès Deboulet et Marie-Antoinette Hily}

\section{QpenEdition}

Journals

Édition électronique

URL : https://journals.openedition.org/echogeo/10944

DOI : 10.4000/echogeo.10944

ISSN : 1963-1197

Éditeur

Pôle de recherche pour l'organisation et la diffusion de l'information géographique (CNRS UMR 8586)

\section{Référence électronique}

Agnès Deboulet et Marie-Antoinette Hily, "Les migrants de Beyrouth », EchoGéo [En ligne], 8| 2009,

mis en ligne le 05 mars 2009, consulté le 31 juillet 2021. URL: http://journals.openedition.org/ echogeo/10944; DOI : https://doi.org/10.4000/echogeo.10944

Ce document a été généré automatiquement le 31 juillet 2021.

EchoGéo est mis à disposition selon les termes de la licence Creative Commons Attribution - Pas d'Utilisation Commerciale - Pas de Modification 4.0 International (CC BY-NC-ND) 


\title{
Les migrants de Beyrouth
}

\author{
Emplois à bon marché et zones refuges
}

Agnès Deboulet et Marie-Antoinette Hily

1 La plupart des grandes métropoles du monde arabe et sud-méditeranéen sont devenues ces dernières années des réceptrices de migrants dont les durées de séjours dépendent de contrats temporaires passés avec des employeurs. Mais nombre d'entre eux ne repartent pas et tentent de trouver du travail dans les secteurs les moins bien payés de l'économie. Qu'ils soient en situation régulière ou non, nombre de ces migrants récemment arrivés et dits " temporaires » ou « en transit ", mais dont certains sont là depuis une dizaine d'années, ont fait leur entrée sur le marché du travail et sont maintenant visibles dans des quartiers périphériques où il est plus facile de trouver à se loger. C'est dans la banlieue de Bourj-Hammoud à l'est de Beyrouth et dans les quartiers sud de Jnah et de Ouzaï, à partir d'enquêtes de terrain, que seront décrites des situations migratoires qui sans être généralisables n'en sont pas moins exemplaires de la place de cette main-d'œuvre immigrée en grande partie féminisée.

\section{Introduction}

2 Si le Liban a connu une forte émigration notamment dans les années de guerre (1975-1990) avec plus de 400000 libanais qui ont quitté le pays dès le début des hostilités, touchant $15 \%$ de la population, il a aussi enregistré un apport de maind'oeuvre important. Les migrations régionales arabes favorisées par l'ouverture des frontières entre la Syrie et le Liban de 1990 à 2005 se composaient majoritairement de Syriens évalués entre 300000 et $500000^{1}$ et d'Égyptiens arrivés en 1997 et dont la situation a été régularisée. Outre ces migrants une part non-estimée est composée de Soudanais qui disposent pour un tiers environ de statut de réfugié ${ }^{2}$ et d'hommes issus de toutes les minorités circulantes du Proche-Orient souvent dépourvus de droits nationaux et issus de migrations forcées ${ }^{3}$. Une autre vague migratoire caractérise les migrations au Liban, celle en provenance des Philippines, du Sri Lanka, de l'Inde et de l'Éthiopie. Selon les statistiques du Ministère du travail ${ }^{4}$ le Liban aurait reçu 120000 travailleurs étrangers entre 1990 et 2005 en provenance de l'Ethiopie, des Philippines, 
du Sri Lanka et de l'Inde. Pour la seule année 2004 les travailleurs étrangers officiellement enregistrés venaient principalement du Sri Lanka (34 972), de l'Ethiopie (21 573), des Philippines (16 471) et d'Inde (5 570).

3 Aujourd'hui sur une population estimée entre 3,5 à 4 millions de résidents dans tout le Liban, un quart de la population active serait composée de travailleurs migrants toutes nationalités confondues, soit un total estimé entre 600000 et 800000 personnes (Clochard et Doraï, 2006 ; Jureidini, 2003). Mais ces données qui ne sont qu'indicatrices des tendances de la migration au Liban ne comptabilisent évidemment pas le volume des personnes non officiellement déclarées ni la répartition par sexe. Notamment elles ne rendent pas compte du fait que ce paysage migratoire est marqué par l'importance de la population féminine. Elle représenterait selon Jureidini (2003) $90 \%$ de l'ensemble des migrants, Syriens exceptés ${ }^{5}$. Bien que nous ne disposions pas de statistiques fiables, il est toutefois possible de mettre en évidence le remarquable apport de cette maind'œuvre à l'économie du pays.

D'autres caractéristiques singulières de ces migrations doivent aussi être mentionnées. D'une part les carrières migratoires sont très significativement liées à la division entre travailleurs arabes et non arabes et à la division sexuée du travail (Bret, 2007). Cette dernière recoupe aussi à la fois des distinctions homme/femme, musulmans/chrétiens et autres religions asiatiques. Par ailleurs les contingents de travailleurs " légaux " importés dans le secteur des services fluctuent en fonction de la nature des rapports diplomatiques entre les Etats et de la conjoncture internationale. Ainsi, l'émigration à partir des Philippines a t-elle été freinée, le Liban étant considéré comme peu sûr pour leurs ressortissantes en raison des nombreux cas de maltraitance répertoriés.

\section{Des situations migratoires héritées de la législation et des spécificités de la demande d'emplois}

5 L'obligation de disposer d'un visa pour pouvoir entrer en tant que travailleur étranger sur le sol libanais est contrôlée par la Direction de la Sûreté générale. Celle-ci accorde des visas pour une période de six mois renouvelable six mois. Elle accorde des cartes de séjours temporaires d'une durée d'un an ou de trois ans renouvelables. Mais le pilier de ces dispositifs repose sur la personnalité juridique d'un garant ou Kafil ${ }^{6}$, que le travailleur étranger soit salarié ou qu'il exerce une profession indépendante.

6 En outre tout candidat à un emploi au Liban doit obtenir une autorisation du Ministère du travail et des Affaires Sociales et posséder un contrat de travail d'un an renouvelable n'autorisant pas le travailleur à changer d'emploi ni à quitter le pays sans l'autorisation explicite du kafil. Dans de nombreux cas, ce dernier se voit confier le passeport du candidat à l'immigration. Les procédures d'obtention des permis de travail sont coûteuses et l'employeur doit justifier l'embauche d'un travailleur étranger en lieu et place d'un Libanais. Muni d'une carte blanche de résidence et d'une carte rouge de travail le travailleur sera durant sa période d'emploi sous la responsabilité de son garant (encore appelé sponsor): l'employeur ou le responsable d'une agence de recrutement par exemple, ce qui est souvent le cas dans le secteur de la domesticité. Mais dans la réalité nombre de travailleurs et travailleuses échappent à cette législation et se retrouvent «sans papiers » soit parce qu'ils prolongent leur séjour au-delà du 
temps réglementaire, soit parce qu'ils fuient leurs employeurs, soit enfin parce qu'ils sont entrés sur le territoire libanais irrégulièrement.

7 A ces éléments législatifs viennent donc s'ajuster des figures de migrants d'où émergent deux traits principaux :

- Premier cas de figure : la filière migratoire dominante, est surtout constituée de Syriens célibataires. Employés dans le bâtiment, les services et le secteur agricole, ils maintiennent des liens réguliers avec leur pays. Mais les travailleurs clandestins semblent compter une part non négligeable de réfugiés de fait, kurdes de Syrie ou de Turquie notamment, syriens menacés politiquement. Pour ces derniers, le maintien des liens avec la famille et les proches est plus problématique, le Liban jouant alors le rôle de pays de transit avant une migration risquée ou hypothétique en Europe (Clochard et Doraï, 2006) ou vers les pays du Golfe. Devant les difficultés à atteindre les pays européens, nombre de migrants ne peuvent faire autrement que de travailler sur place en attendant l'opportunité d'un départ vers des régions dont ils pensent que les ressources sont plus rentables. Dans cette logique il apparaît quasiment impossible de faire la distinction entre pays de transit et pays de destination.

- Second cas de figure: l'importation d'une main-d'œuvre féminine comme domestiques et gardes d'enfants à domicile (parfois de personnes âgées) est très importante depuis la fin de la guerre (1990). Ces salariées reflètent directement une hiérarchie explicite basée sur la nationalité d'origine, censée incarner des dispositions différenciées à s'occuper d'une maison mais aussi un niveau de formation initial distinct. Issues de pays en développement et en forte crise économique ou politique, ces femmes transfèrent leur savoir-faire domestique et leur capacité d'attention (care) et d'amour notamment maternel (Ehrenreich et Russell Hochschild, 2002) à des ménages fortunés du «premier monde ", mais aussi - de plus en plus fréquemment -, au sein des classes moyennes. Cette main-d'œuvre est « à demeure » chez l'employeur, souvent mal payée (le salaire étant inférieur au revenu minimum libanais) et sous contrôle du garant. Dans ce cas, l'employée ne bénéficie dans le meilleur des cas que d'une journée de repos par semaine et se charge de toutes les taches ménagères en plus de la garde des enfants. D'autres situations d'emploi (légal ou non) permettent à certaines femmes de vivre de façon autonome ${ }^{7}$, généralement à plusieurs dans des appartements qu'elles louent souvent au prix fort et dans des zones dégradées où se retrouve une population d'hommes et de femmes aux parcours complexes. En situation irrégulière pour la très grande majorité, ces migrantes sont tournées d'abord vers la recherche d'une occupation même faiblement rémunérée. ${ }^{8}$

10 Ces deux segments complémentaires du marché de travail immigré se scindent en plusieurs sous-segments. Le premier, déjà évoqué, reprend une division classique de l'emploi de migrants dans le monde, qui recoupe employés légaux-illégaux, travail formel et informel. Bien que ces dernières oppositions soient critiquées pour leur formalisme qui ne rend pas compte de la diversité des situations et de leur imbrication, la grille de lecture entre emploi formel et informel continue à être utilisée, faute de mieux. Toutefois on doit encore souligner la porosité entre ces frontières, en rappelant que clandestinité des travailleurs migrants et par voie de conséquence l' "informalité » du travail sont plutôt la règle que l'exception. Dans ce cas de figure, la protection dépend à la fois des relations de proximité (appartenance nationale ou régionale, liens familiaux), principal support des rapports de confiance. Le second sous-segment concerne des emplois féminins de travailleuses domestiques dont la position dans 
l'économie internationale interpelle de plus en plus les chercheurs en raison de la féminisation des migrations (aujourd'hui dominante), et de la mise en place de rapports de subordination partiellement déterritorialisés.

\section{Des migrants dans les quartiers périphériques et dans des zones d'habitat précaire non légalisé}

11 Bourj-Hammoud ${ }^{9}$, banlieue considérée comme majoritairement arménienne ${ }^{10}$, à l'est de la capitale accueille selon des estimations non officielles plus d'un millier de migrants sri-Lankais, philippins, bangladais, indiens, éthiopiens, égyptiens, ghanéens, syriens, soudanais, kurdes et béninois. Ces personnes ont trouvé dans cet espace urbain aux immeubles à plusieurs étages et à l'habitat serré et dense des occasions, qu'elles paient chèrement, de se loger dans des appartements vétustes et mal entretenus. Certains $y$ travaillent comme employés dans des magasins (coursiers), des restaurants (serveurs, cuisiniers, plongeurs, nettoyage...), des petites industries (charcuterie, fabrication de la bière locale, fabrique de fours à pain, industrie du sac plastique) ou encore des fabriques de chaussures, de vêtements et enfin des garages. Quelques-uns, une faible minorité, ont développé un petit commerce de restauration-épicerie, de ventes de vêtements et tissus, de cassettes de chanteurs et musique, indiens. Les femmes qui ne travaillent pas dans le quartier font des ménages dans des familles aisées beyrouthines; les hommes rejoignant le personnel sous-payé des restaurants et des entreprises de bâtiments.

Nombre de ces migrants sont en situation illégale et vivent dans la précarité, dans la peur du contrôle. Et pourtant c'est dans cette incertitude quotidienne que s'organise une vie sociale où ils tentent de conjuguer nécessité de la discrétion, évitement des «locaux/nationaux» et recherche de soutien et de solidarités pour faire face aux difficultés quotidiennes, à la solitude et à l'éloignement du pays natal.

De «nouveaux » commerçants se sont néanmoins installés dans le quartier à la faveur de contrats informels passés avec des propriétaires libanais et les signes de leur appartenance sont devenus bien visibles. Aujourd'hui, une dizaine de ces magasinsrestaurants, sri lankais et indiens peuvent offrir des produits qui répondent aux demandes des migrants : épices, primeurs, petite épicerie, restauration et bijouterie. La rue centrale du quartier (la rue d'Arménie) devient désormais le dimanche un espace « cosmopolite » où les migrants «beyrouthins » se rencontrent. À l'heure du repas les restaurants indiens, sri lankais et philippins accueillent ceux et celles qui ont assisté à une messe dans une salle transformée en église à l'étage d'un centre associatif géré par des sœurs libanaises ou encore les Indiens qui se sont retrouvés dans un espace de réunion pour pratiquer leur religion, le bouddhisme. En recréant des espaces de sociabilité passagers ces migrants tentent de se construire un «milieu » où ils se reconnaissent dans le partage d'un moment et d'un espace qui permettent la rencontre même éphémère.

De nombreuses poches d'habitat précaire, non légalisé ${ }^{11}$, issu du squat foncier ou du lotissement clandestin, recueillent également des migrants issus de ces nombreux points de convergence mondiaux, dans les quartiers sud ou est de la capitale. Pour ce qui est des quartiers sud, composant en tout ou partie ce que les beyrouthins nomment la « banlieue » (dahiye) de Beyrouth (Clerc, 2008 ; Deboulet, 2008 ; Fawaz, 2004), certains sont dorénavant peuplés d'une population migrante aussi nombreuse qu'hétérogène. 
Jouxtant les camps de réfugiés qui sont eux le refuge de nombreux travailleurs syriens, la perception des quartiers de Jnah et d'Ouzaï comme des « bastions » shi'ites ne résiste pas à l'examen. En effet, certains sous-secteurs contiennent une population migrante très importante, quoique non comptabilisée. Nous pouvons l'estimer d'un tiers à la moitié des résidents, selon les secteurs. Une des particularités les plus saillantes de cet habitant migrant est sa double spécialisation: en effet, les "étrangers » dans ces quartiers sont dans l'ensemble presque toujours célibataires ou sans enfant au Liban, et vivent dans des logements non familiaux: chambres occupées à plusieurs, ou deux pièces. En outre, la présence de femmes célibataires (d'Asie et d'Afrique) et d'hommes célibataires (du Proche-orient) génère une partition de l'espace qui obéit aussi aux règles d'évitement et de civilité entre migrants par ailleurs issus d'univers géographiques radicalement éloignés.

L'arrivée dans ces quartiers génère une double précarité. Car ces migrants sont souvent privés de papiers, dénués de toute forme de citoyenneté comme de possibilité de retour. Ils sont également particulièrement dénués d'autres formes de protection, locative ou résidentielle, résidant dans des quartiers vivant sous la menace de l'éviction. En revanche, la faible présence étatique dans des secteurs contrôlés par le Hezbollah ou Amal leur garantit une certaine sécurité et évite de subir les rafles qui sont par contre devenues courantes dès que l'on quitte la dahiye. A l'évidence les formes de circulation dans la ville sont fortement affectées par ce double standard d'insécurité. $\mathrm{Au}$ demeurant, nombreux sont les migrants rencontrés qui ignorent l'existence des ONGs qui peuvent les accompagner dans la recherche de documents d'identité dont ils ont été dépossédés où leur venir en aide quand ils se retrouvent démunis de tout.

\section{Conclusion}

16 A Beyrouth les migrants trouvent des lieux à investir, sans se les approprier pleinement pour autantdans la mesure où ils vivent souvent leur installation comme provisoire et se sentent peu légitimés voire faiblement acceptés par les locaux. Ils mobilisent en outre des micro-réseaux pour résister ou s'adapter à l'environnement urbain. Qu'ils ne soient que de passage (en «transit», notion plus qu'incertaine) ou qu'ils se stabilisent, par leur présence, leur visibilité et leurs pratiques, ils donnent du contenu au mot «mondialisation». Des espaces citadins périphériques voient donc de nouvelles populations s'installer pour un temps plus ou moins long selon les possibilités ou les empêchements de partir et qui malgré les contraintes qu'elles subissent tentent de se créer un espace de vie qui repose sur les liens qu'ils nouent entre compatriotes mais aussi avec les segments choisis de migrants jugés proches ou compatibles.

Bourj-Hammoud comme Jnah et Ouzaï accueillent ainsi une nouvelle main-d'œuvre pauvre et privée de droits qui désormais fait partie de la réalité économique du Liban. Elle peut venir modifier le paysage urbain par une présence plus longue et s'inscrire plus durablement dans un pays qui sans le reconnaître officiellement n'ignore pas le rôle qu'elle joue sur le marché du travail, en alimentant en particulier un secteur des services à bas prix qui participe largement à la qualité de vie des classes moyennes supérieures et des catégories dominantes. 


\section{BIBLIOGRAPHIE}

Bergem, K V., 2006, "The Role of the State in the in-Migration of Domestic Workers to Jordan and the GCC Countries", in De Bel-Air Françoise (sous la dir de) (2006) Migration et politique au MoyenOrient, IFPO, Beyrouth, pp 61-79.

Berthomière, W. et Hily M-A., 2006, « Décrire les migrations internationales. Les expériences de la co-présence », Revue Européenne des Migrations Internationales, 22-2, p. 67-81.

Berthomière, W., Doraï M. et de Tapia S. (sous la dir. de), 2003. Moyen-Orient : mutations récentes d'un carrefour migratoire, Revue Européenne des Migrations Internationales,19-3.

Bret J., 2007, Circulations transnationales et travail disqualifié au Moyen-Orient. Les travailleurs non arabes au Liban, Hommes et Migrations, n 1266, p. 96-107.

Clerc V., 2008, Les quartiers irréguliers de Beyrouth, une histoire des enjeux fonciers et urbanistiques dans la banlieue sud, Institut Français du Proche Orient, Beyrouth.

Cobban H., 1985, The Making of Modern Lebanon. London : Hutchison and Co. Publishers Ltd.

Clochard O. et Doraï M. K., 2006, Non-Palestinian Refugess in Lebanon. From Asylum Seekers to Illegal Migrants, in De Bel-Air Françoise (sous la dir de) (2006) Migration et politique au MoyenOrient, IFPO, Beyrouth, p. 127-143.

Deboulet A, 2008, « Ethiopiennes, Philippines et soudanais : voisinages migrants et confrontation aux sociétés d'accueil au Liban ", Studi Emigrazione, Roma, n 172, oct-déc. EhrenreichB., Russell HochschildA., Global woman : Nannies, maids, and sex workers of the new economy, New York, Metropolitan books, 2002.

Fawaz M., 2004, Strategizing for Housing: An investigation of the production and regulation of low-income housing in the suburbs of Beirut. Unpublished PhD Dissertation, Cambridge, MA: Massachusetts Institute of Technology.

HILY M., 2009, « Migrants dans une banlieue beyrouthine », Maghreb-Machrek, printemps 2009 (à paraître).

Jaber H et Métral F., 2005, Mondes en mouvements. Migrants et migrations au Moyen-Orient au tournant du XXIe siècle, IFPO, Beyrouth.

Jureidini R., 2003, L'échec de la protection de l'Etat : les domestiques étrangers au Liban, Revue Européenne des Migrations Internationales, 19-3, p. 95-128.

Picard E., 2001. « Les Syriens, l'envers du décor », in Tabet J., ( sous la dir.), Beyrouth, la brûlure des rêves, Paris, Autrement, p. 92-102

\section{NOTES}

1. En 2005 plusieurs milliers de travailleurs syriens quittent le Liban après l'assassinat du Premier Ministre Rafiq Hariri et le retrait des forces armées syriennes (Voir Picard, 2001). Aujourd'hui un grand nombre de travailleurs sont repartis dans leur pays alors que d'autres bien visibles dans la capitale travaillent dans les restaurants, dans les hôpitaux, les usines de confection, la mécanique. Il faut préciser d'emblée sur ce point comme sur la comptabilité des flux entrants l'absence totale de fiabilité des statistiques sur les migrants.

2. La demande d'asile politique a doublé entre 1996 et 2000 (Clochard et Doraï, 2006). 
3. Kurdes, Irakiens, Soudanais du sud ont fui des contextes de guerre et de privation en passant la frontière clandestinement par la Syrie. Tous occupent des emplois manuels, qu'ils soient qualifiés ou non, les Egyptiens et les Soudanais étant plus fréquemment employés dans les services (garages, restauration).

4. Chiffres fournis par S. Menhem (thèse en cours: Les nouveaux immigrés au Liban: vers une ethnicisation du marché de l'emploi ?)

5. Soit 90000 femmes essentiellement non arabes (Jureidini, 2003).

6. Le kafil est l'intermédiaire, légalement reconnu par les autorités, entre les services officiels des administrations et les travailleurs étrangers.

7. Appelées "travailleuses free lance " elles trouvent à s'employer à l'heure pour faire des ménages.

8. Ces différentes pratiques à l'égard du droit fragilisent les migrants et les maintiennent dans des situations où toutes actions éventuelles contre des abus ou des manquements aux droits de la personne est sans cadre juridique légal. En l'absence de droits dont les institutions étatiques seraient le garant, nombre de rapports émanant d'ONG libanaises ont entrepris de dénoncer les conditions de travail dont sont victimes ces travailleurs (Jureidini, 2003 ; Bergem, 2006). Les principales instances de recours sont en effet les ONG, dont le Comité pastoral des Migrants Afroasiatiques dirigé par Caritas, et dans une moindre mesure les ambassades. Ce sont d'ailleurs ces ONG qui ont contribué à rendre visible cette population en se manifestant sur la scène publique lors du raz-de-marée de 2004 qui ravagea les côtes de l'Indonésie, du Sri Lanka, des Philippines et de l'Inde, pays d'origine des migrants .

9. Voir Hily (2009)

10. Ce que les statistiques non actualisées ne peuvent rendre compte

11. Voir Deboulet (2008)

\section{RÉSUMÉS}

Depuis la fin de la guerre (1990) l'importation d'une main-d'œuvre peu qualifiée majoritairement féminine est devenue massive au Liban. Qu'ils soient en situation régulière ou non, des migrants non arabes dits «temporaires » ou « en transit », mais dont certains sont là depuis une dizaine d'années, ont fait leur entrée sur le marché du travail et tentent de s'inscrire, pour nombre d'entre eux, dans un "milieu » aux identités multiples et conflictuelles dans des quartiers périphériques où il est plus facile de trouver à se loger. En s'appuyant sur des enquêtes de terrain menées dans la banlieue de Bourj-Hammoud à l'est de Beyrouth et dans les quartiers sud de Jnah et de Ouzaï, les auteures décrivent des situations migratoires qui sans être généralisables n'en sont pas moins exemplaires de la place de cette main-d'œuvre immigrée.

Since the end of the war, in 1990, unqualified, mostly feminine, workers have been massively entering Lebanon. Whether they have regular registration or not, non-Arab, so-called 'temporary', or 'transit' migrants have entered the labour market, but some of them have been there for about ten years. Many try to join the mixed, full of conflicts environment of peripheral districts, in which it is easier to find housing. Using fieldwork they have been conducting in the Borj-Hamoud suburb, in the east of Beirut, and Jnah and Ouzaï, in the south, the authors describe migratory situations which, although they cannot be applied generally, are nevertheless a good example of the place held by these immigrant 
INDEX

Mots-clés : Beyrouth, situations migratoires, main-d'œuvre immigrée

Keywords : Beirut, Migratory situations, Foreign workers

\section{AUTEURS}

\section{AGNÈS DEBOULET}

Agnès Deboulet est professeure à l'Ecole nationale supérieure d'architecture Paris-la Villette, UMR AUS.

\section{MARIE-ANTOINETTE HILY}

Marie-Antoinette Hily est chargée de recherches, Université de Poitiers, CNRS/Migrinter UMR 6588. 